\title{
Application Of Prolene Hernia System For Umbilical Hernia In Patients With Mild To Moderate Ascites
}

\author{
Mohamed Hassan M.D ${ }^{1}$, Mohamed Salah M.D. ${ }^{2}$ \\ Department of general surgery ${ }^{1}$, Cairo university, Department of tropical medicine ${ }^{2}$, \\ Cairo University.
}

\begin{abstract}
Background: In 1998, the Prolene Hernia System (PHS) mesh, consisting of an onlay and an underlay patch attached with a connector, was introduced as an option for tension-free open repair of inguinal hernias combining the benefits of a posterior and anterior repair from an open approach.

Our objective was to evaluate the PHS mesh repair of umbilical hernia in ascetics with liver cirrhosis.

Patients and methods: non randomized prospective study included 24 cases with mild or moderate ascites , cirrhotics, with small umbilical hernia were subjected to elective repair using prolene hernia system(PHS) under local anathesia, patients were followed up for one and half years.

Results: no mortality, one case of bleeding varices after 9 months respond to injection, transient ascetic leak in 6 cases, one case of wound infection respond to antibiotics and dressing and no hernia recurrence.

Conclusion: Prolene hernia system is easily applicable and effective method for repair of umbilical hernia with ascites.
\end{abstract}

\section{Introduction}

Ascites is caused by cirrhosis in $75 \%$ of cases, malignancy in $10 \%$, and cardiac failure in 5\%; other causes account for the remaining $10 \%$. The formation of ascites in cirrhosis is due to a combination of abnormalities in both renal function and portal and splanchnic circulation. The main pathogenic factor is sodium retention. About half of patients with cirrhosis develop ascites during 10 years of observation (Krige and Beckingham, 2001). Umbilical hernias can be found in up to $20 \%$ of cirrhotic patients with ascites (Maniatis and Hunt, 2001). Spontaneous rupture of umbilical hernias in these patients is an infrequent event, associated with a $50 \%$ mortality rate with supportive care and a $10-20 \%$ mortality rate after urgent surgical repair (Kirkpatrick and Schuber, 1988 ;MacLellan et al, 1990 ; Maniatis and Hunt, 2001)

Umbilical hernia in cirrhotic patients with uncontrolled ascites was associated with significant mortality (8.3\%) and morbidity (16.6\%) and a significantly greater incidence of recurrence $(16.6 \%)$ (Leonetti et al.,1984)

The use of a mesh plug in hernia repair is not a new concept with previous investigators yielding consistently excellent results in the repair of femoral and inguinal hernias, mesh plug repair can be performed with minimal postoperative complications, low recurrence rate, minimal post-operative pain and achieving excellent patient satisfaction (Sinha and Keit, 2004).

The PHS is made of polypropylene mesh and consists of 3 parts: an onlay patch, a connector, and underlay patch (Cafer et al. ,2005) radical operation using PHS is useful for adult umbilical hernia (Makoto et al., 2002).

\section{Patient and methods}

Our study includes 24 cases of noncomplicated umbilical hernia with small defect $(2$ to $4 \mathrm{~cm})$ in patients with mild to 


\section{Mohamed Hassan \& Mohamed Salah}

moderate ascites attending the tropical medicine department ,cairo university hospitals from May 2005 to May 2007.

All patients were subjected to history taking, general and abdominal examinations, laboratory tests include complete blood picture, liver and kidney function tests, bleeding profiles and serum albumin. Radiological investigations included abdominal and pelvic ultrasonography and electrocardiogram. Also upper GIT endoscopy and analysis of ascetic fluid where done.

Exclusion criteria include abdominal malignancy, tense ascites, child-C patients, impending rupture hernia, spontaneous bacterial peritonitis and refractory ascites.

Preoperative preparation for management of ascites started with salt restriction $(88 \mathrm{mmol} / \mathrm{day})$ and oral diuretics (100-400mg spironolactone) and 40-160mg frusemide as single morning doses. Paracentesis in diuretic resistant cases ( 2 cases) with albumin infusion $10 \mathrm{~g} / \mathrm{liter}$ of paracentesis above 5 liter. Follow up of the treatment was done by daily body weight, abdominal girth, 24 hours urinary output, serum sodium \& potassium and 24 hours urinary sodium execretion.

All operations were conducted under local anathesia with or without sedation with lidocaine hydrochloride dose up to 5 $\mathrm{mg} / \mathrm{Kg}$ and $7 \mathrm{mg} / \mathrm{Kg}$ when 1:200,000 epinephrine was added in non cardiac patients.

Elliptical skin incision, conventional approach was performed until the step of hernia sac reduction. The peritoneum was separated from the posterior aponeurosis of the rectus abdominis muscle to create enough space within the preperitoneal area for underlay patch of the PHS. Any laceration of the peritoneum was closed or omentum reposed to avoid any contact of the mesh with intestine. The underlay patch of the PHS was placed in the preperitoneal space without suture. The edge of the aponeurosis was closed with interrupted sutures, leaving a hole at the umbilical ring where the connector was passed through. Onlay patch was fixed to the anterior rectus sheath with polypropylene suture (Prolene, $2 / 0$ ). Umbilicus was sutured to the connector with polypropylene suture. Subcutaneous suction drain was used in all cases removed after 24 hours with the drain site closed by vertical mattress suture and collodion application.

Postoperative care include intravenous $3^{\text {rd }}$ generation cephalosporine for 7 days then oral forms for 15 days. All patients received a routine intramuscular dose of $50 \mathrm{mg}$ pethidine $\mathrm{HCl}$ on the evening of the operation day. A 75-mg dose of diclophenac sodium was given as needed.

The patients were discharged from the hospital at the $10^{\text {th }}$ day, the patients were followed-up postoperatively for the first week, then 1 month later, and finally every 6 months for one and half years.

\section{Results}

This study was conducted on 24 patients with mild to moderate ascites having umbilical hernia with male to female ratio 10:14, age range 33:59 and follows up ranging from 18 to 24 months with an average of 21 months.

Operative time range from 35 to 50 minutes, local anathesia alone in 18 patients, sedation was needed in 6 patients. The mesh was easily applied but the peritoneum was opened in 4 cases, in 3 cases repaired successfully and the omentum was used in the $4^{\text {th }}$ case. Suction drain was inserted in all cases.

Postoperative ascites control was achieved with continuation of preoperative regimen. Paracentesis with albumin infusion was required in 6 cases.

No mortality or recurrence in our study. There were early minor postoperative complications in the form of transient ascetic leak in 6 cases respond well to drain site closure, abdominal paracentesis and collodion application. One case of wound infection responds to IV antibiotics \& resolves within 2 weeks. One case of bleeding oesophageal varices after 9 months responds to injection sclerotherapy. 
Application Of Prolene Hernia System For............

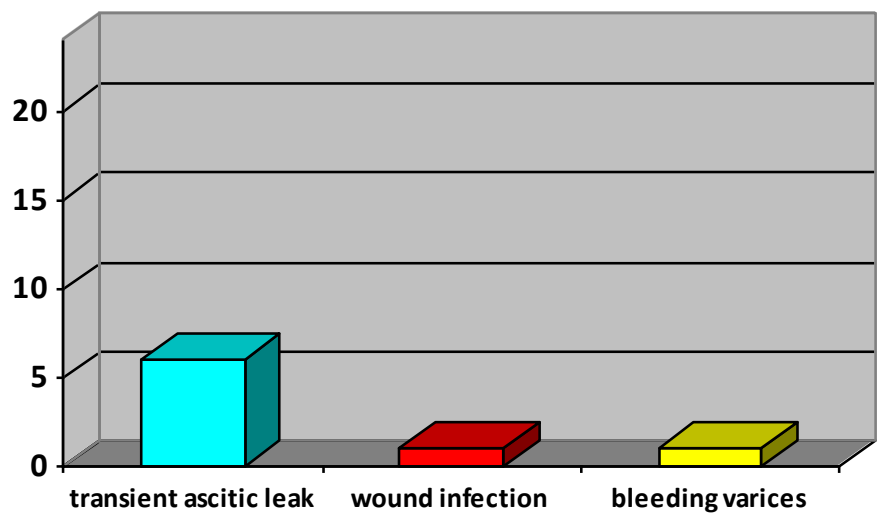

$\square$ transient ascitic leak $\square$ wound infection

$\square$ bleeding varices

Fig. 1: postoperative complications

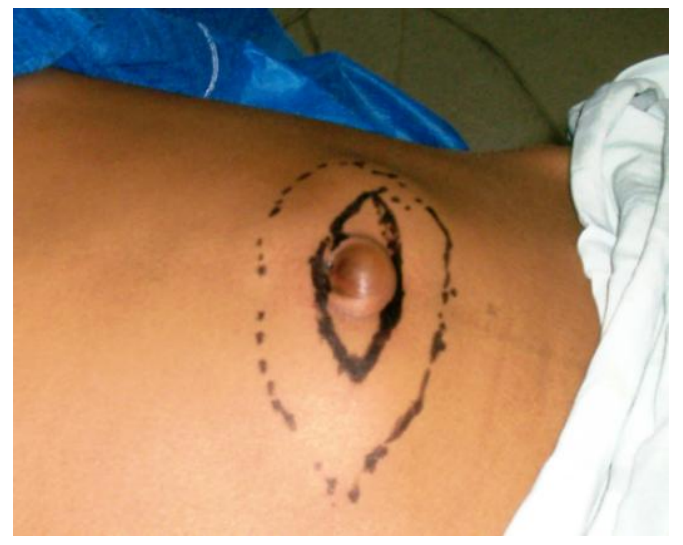

Fig. 2: Area of local anathesia.

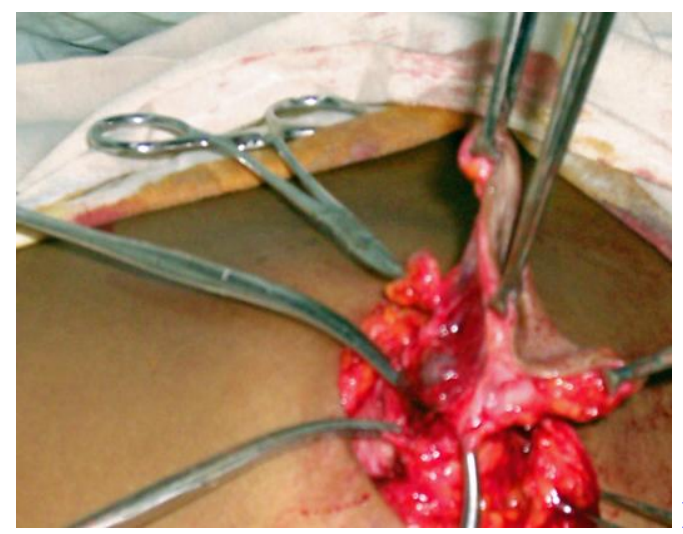

Fig. 3: Dissection of the sac

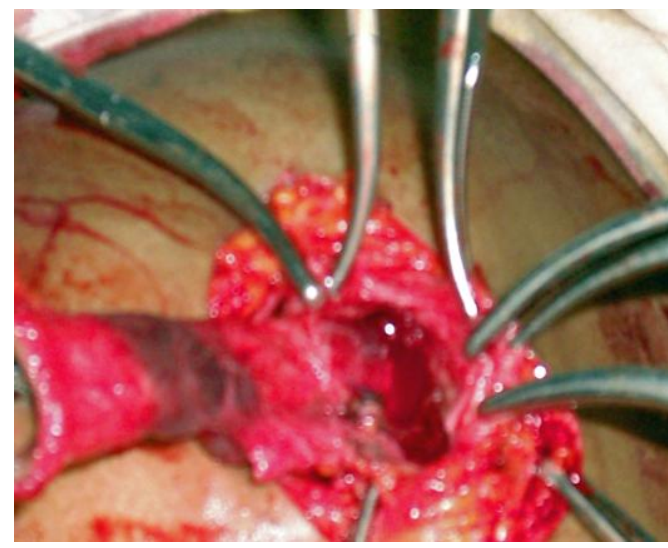

Fig. 4: Creation of preperitoneal space 


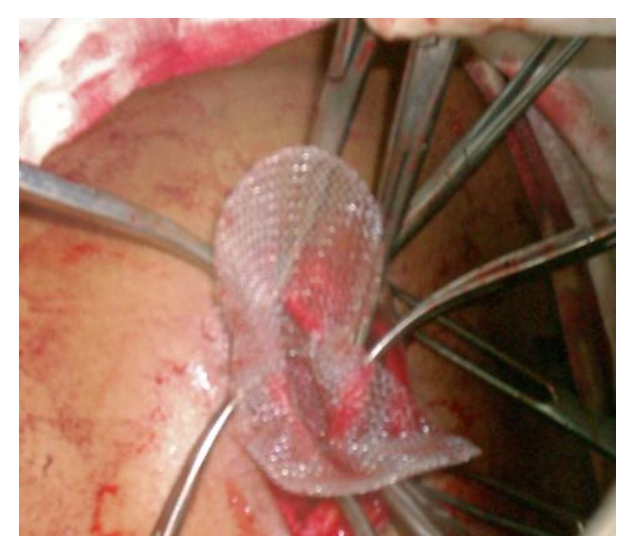

Fig. 5: Application of the PHS

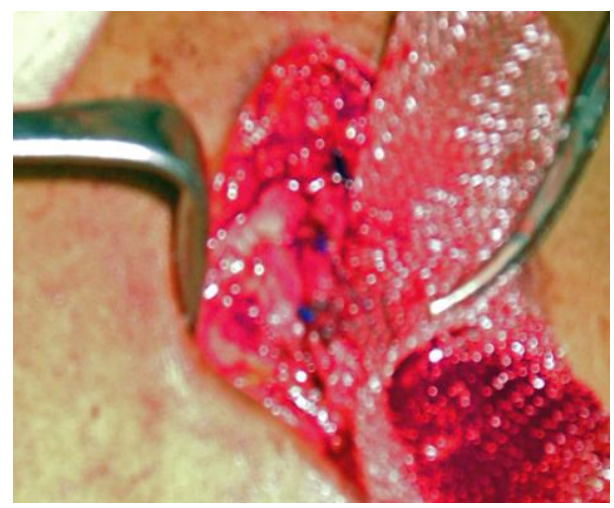

Fig. 6: Suturing the connector to the defect

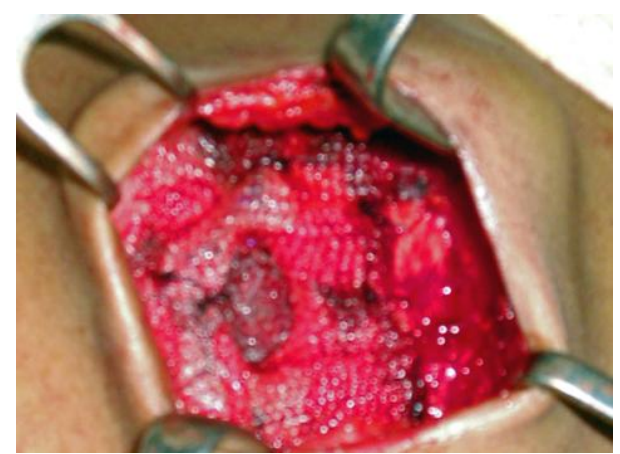

Fig. 7: Complete mesh application

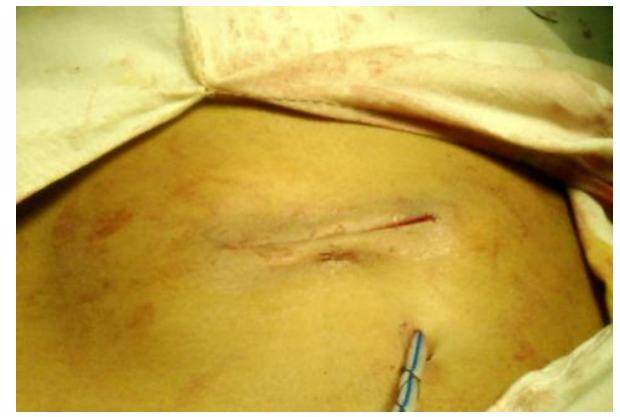

Fig. 8: Closure with subcutaneous drain 


\section{Discussion}

The PHS mesh method for inguinal hernia repair was associated with a lower recurrence rate as well as fewer complications when compared with the Lichtenstein onlay mesh repair technique. These low recurrence rates may stem from the complete coverage of the myopectineal orifice and flexibility of the PHS mesh (Samir et al. ,2007).

Prosthetic repair has become the standard method for inguinal hernia and has excellent results. The question remains of whether the mesh technique could also improve results for umbilical defects, prosthetic repair could become the standard treatment for primary umbilical hernia in adults (Arroyo et al., 2001).

The outcomes of the PHS for umbilical hernia repairs appear to be better for selected patients than the other techniques in terms of minimal postoperative pain and less requirement of analgesics. It also offers an advantage of combination of 3 techniques, such as Stoppa-plug-onlay mesh repair, against recurrence (Cafer et al., 2005). In our study there is no recurrence with minor postoperative complications.

Operative management is indicated for umbilical hernias to avoid rupture, although recent practice avoided elective repair, it is associated with only a $2 \%$ mortality rate. In contrast, emergent repair in cases of rupture, leak, incarceration, and strangulation is associated with a $14 \%$ mortality rate (Maniatis et al .,1990).

Elective repair of uncomplicated umbilical hernias in cirrhotic patients with ascites might obviate the risky emergency operations for their complications (Abbas $e t$ al.,2002).

Our study was conducted for patients with mild to moderate ascites as cirrhotic patients with massive ascites who require an umbilical herniorrhaphy preferably should undergo peritoneovenous shunting or Transjugular Intrahepatic Portovenous Shunt (TIPS) before repair of the hernia (Leonetti et al.,1984).

Our operative time range from 35 to 50 minutes which is comparable to that reported by Arroyo et al. (2001) and Sinha et al. (2004).

Closure of the hernial defect should not take place at the expense of increasing the stretch on the already exhausted stretched aponeurosis. The hernial orifice should be filled or closed without any additional tension(Sinha and Keit ,2004). These fundamental principles appear to be satisfied by suturing the edge of the defect to the connecter in our series.

\section{Conclusion}

Umbilical hernia repair in patients with mild to moderate ascites using prolene hernia system under local anathesia is a safe effective procedure with less postoperative complications and recurrence rate, obviate the high morbidity and mortality of emergency operation.

\section{Reference}

1- Krige J E J and Beckingham I J (2001): Clinical review - ABC of diseases of liver, pancreas, and biliary system. BMJ., 322:416-418.

2- Maniatis A G and Hunt CM (1995): Therapy for spontaneous umbilical hernia rupture. Am. J. Gastroenterol.., $90: 310-312$.

3- MacLellan DG, Watson K J and Farrow HC (1990): Spontaneous paracentesis following rupture of an umbilical hernia. Aust. N. Z. J. Surg., 60 : 555-556.

4- Kirkpatrick $\mathbf{S}$ and Schubert $\mathbf{T}$ (1988): Umbilical hernia rupture in cirrhotics with ascites. Dig. Dis. Sci., 33 : 762-765.

5- Leonetti JP, Aranha GV, Wilkinson WA, Stanley M, Greenlee HB (1984): Umbilical herniorrhaphy in cirrho-tic patients. Arch. Surg., 119(4):442-5.

6- Sinha SN and Keit T (2004): Mesh plug repair for paraumbilical hernia. Surg. J. R. 
Coll. Surg. Edinb. Irel., 2 : 99102.

7- Cafer P, Adem D, Gokhan S, Mehmet B, Kenan $E$ and Kayhan O (2005): Umbilical hernia repair with the prolene hernia system. Am. J. Surg.,190(1): 61-64.

8- Okazaki M, Shinozaki K, Terado $M$

and Yamamura J (2002): An operation case of umbilical hernia with Prolene Hernia System. Surg. Therap. , 87(1):112115.

9- Samir S A, Sasi Y BA, Ahmad M S, Charles F B, Daniel A and David $H$ B (2007): Improved outcomes with the Prolene Hernia System mesh compared with the time-honored Lichtenstein onlay mesh repair for inguinal hernia repair. Am. J. Surg., 193( 6): 697-701.

10- Arroyo A, Garcia P, Perez F, Andreu J, Candela F.and Calpena R. (2001): Randomized clinical trial comparing suture and mesh repair of umbilical hernia in adults. Br. J. Surg., 88(10): 1321 $-1323$.

11- Abbas M., El-Ghannam M.,Mobarak M. and ElDamarawy M.(2002): Elective mesh cone and cover repair of umbilical hernia in patients with cirrhotic liver and ascites. Egypt. J.Surg. , 21(4): 375-379. 


\section{إستخدام النظام الشبكي البروليني لعلاج الفتق السري في مرضي

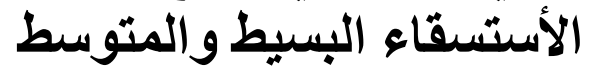

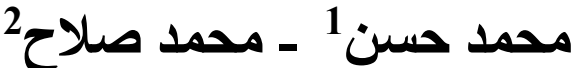

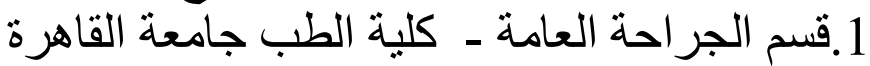

2.قسم الأمر اض المنوطنة - كلية الطب جامعة القاهرة

تم إجر اء هذا البحث علي أربعة و عشرون مريض بالإستسقاء يعانون من الفتق بإنق

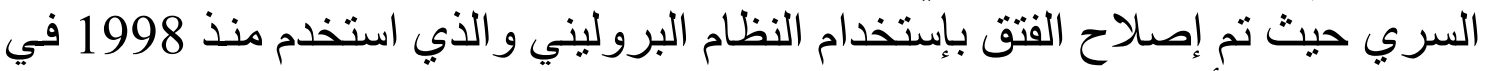

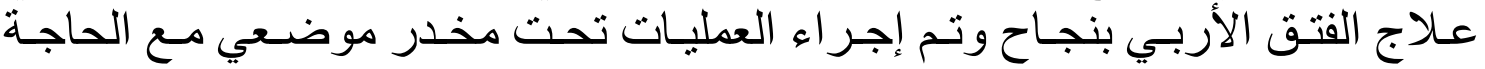

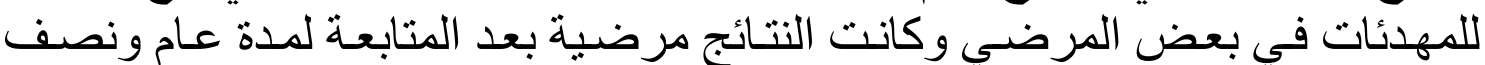

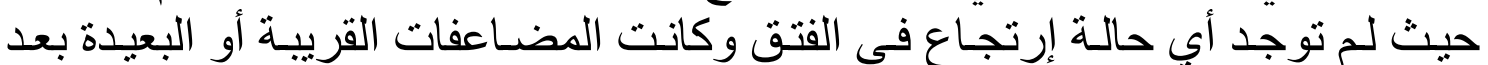
العملية بسيطة وسهل التعامل معها. 